\title{
VON DER RHETORIK ZUR RHETORISCHEN KOMMUNIKATION: EIN TERMINOLOGISCHER UND INHALTLICHER WANDEL
}

\section{Einleitung}

Der Ausdruck Rhetorische Kommunikation wurde in die deutschsprachigen Fachliteratur von Brigitte Frank-Böhringer in ihrem gleichnamigen Buch (1963) eingeführt. Mittlerweile wird dieser Terminus in Kontexten verwendet, die auf rhetorische Aspekte hindeuten. Er taucht sowohl in rhetorischen Ratgebern als auch in wissenschaftlichen empiriach angelegten Rhetorik-Veröffentlichungen auf. Der vorliegende Beitrag hat zum Ziel, die wissenschaftstheoretischen Hintergründe des Begriffs der Rhetorischen Kommunikation näher zu beleuchten, indem auf sprechwissenschaftlich relevante Kommunikationsansätze eingegangen wird. Grundlegend dafür ist eine differenzierte Verwendung des Kommunikationsbegriffs und die handlungstheoretische sowie die ganzheitliche Betrachtung der gesprochenen Sprache. Abschließend wird der praxisbezogene Wert des Begriffs der Rhetorischen Kommunikation hervorgehoben: Sein inhaltlicher Gehalt stellt eine begründbare Grundlage für die Einbeziehung der rhetorischen Inhalte in die Vermittlung des Deutschen als Fremdsprache dar, die jeweils zweckgerichtet und systematisch erfolgen soll. Ausgegangen wird im Folgenden von der Auffassung der Rhetorischen Kommunikation.

\section{Rhetorische Kommunikation — die inhaltliche Besetzung des Begriffs}

\subsection{Eine neue Perspektive der klassischen Begrifflichkeit}

In der antiken Auffassung gilt Rhetorik als ,politisch und ethisch fundiertes Lehrsystem wirksamer öffentlicher Rede“ (Bußmann 1990, S. 648). Demgegenüber rückt mit dem Terminus der Rhetorischen Kommunikation ein umfassendes

* Dr. Beata Grzeszczakowska-Pawlikowska, Universität Łódź, Philologische Fakultät, Lehrstuhl für Deutsche und Angewandte Sprachwissenschaft, 90-236 Łódź, Pomorska 171/173. 
kommunikativ-dialogisches Grundverständnis sozialer Interaktionen ins Bewusstsein. Damit wird auf eine Begrifflichkeit gezielt, die für „die Prozesse des ,Sprechens mit anderen', also Gespräche, und des ,Sprechens zu anderen', also Reden“" (Geißner 1981, S. 153) in gleicher Weise zutreffend ist (vgl. auch ebd., S. 144; Teuchert 1992, S. 67f.; Gutenberg 2001, S. 147). Hervorgehoben wird somit einerseits die aktuelle Dialogizität der Gesprächsrhetorik und andererseits die virtuelle Dialogizität der Rederhetorik (vgl. Neuber 2013, S. 102). Der Begriff der Rhetorischen Kommunikation versteht sich folglich als Weiterentwicklung des herkömmlichen Rhetorikverständnisses. Dennoch wird nach wie vor auf grundlegende Erkenntnisse der klassischen Rhetorik systematisch zurückgegriffen. Auf antiken rhetorischen Leitsätzen, wie etwa Produktionsstadien der Rede, werden z.B. moderne Rede-Trainings aufgebaut. Die neue Bezeichnung wird allerdings zugleich „der an der ,monologischen' Redner- oder Rederhetorik orientierten klassischen und nachklassischen ,Rhetorik““ (Geißner 1981, S. 144) gegenübergestellt und dadurch bewusst u.a. gegen die rhetorische Technik und die daraus resultierende imperative und manipulative Rhetorik abgesetzt (vgl. Gutenberg 1994, S. 25): „Rhetorik als ,Sozialtechonologie“ sollte abgelöst werden durch rhetorische Kommunikation als Verständigungshandlung“ (ders. 2001, S. 146).

\subsection{Rhetorische Kommunikation als Fachgebiet}

Als Fachgebiet (Teilgebiet der Sprechwissenschaft, d.h. der Theorie der mündlichen Kommunikation) beschäftigt sich Rhetorische Kommunikation ,mit den Prozessen handlungsauslösenden Sprechdenkens und Hörverstehens [Sprechund Hörhandelns] in den verschiedensten Gesprächs- und Redeformen" (PabstWeinschenk 2004, S. 101; vgl. auch Geißner 1986a, S. 19), die in den Vordergrund diesbezüglicher Theorien, Forschung und Lehre treten. Aufbauend auf der dialogischen Auffassung der Rhetorischen Kommunikation wird dabei grundsätzlich kein Unterschied zwischen Gespräch und Rede gemacht (vgl. Pabst-Weinschenk ebd., S. 66). Mit anderen Worten: Die Grundform der Rhetorischen Kommunikation ist das Frage-Antwort-Muster, das nicht nur Gesprächen, sondern auch Redeformen zugrunde liegt. Auf der Mikroebene gehören zur Rhetorischen Kommunikation sowohl informative als auch persuasive Akte, da es sowohl bei Gesprächen als auch bei Reden klärende und lösungssuchende (informative) wie auch streitende und entscheidende (persuasive) Verfahren gibt (vgl. Geißner 1986a, S. 132). Die Rhetorische Kommunikation lässt sich nicht zuletzt als linguistisches Handlungsmodell interpretieren, im Rahmen dessen ein aktiver Redner einem reaktiven Hörer gegenüber steht: Der Redner handelt denkend (Vorwissen, Vorurteile), erlebend (Erlebnisfähigkeit, Erfahrungshorizont) und wertend (Wert- und Normsystem der Bezugsgruppe). Der Hörer handelt entsprechend mit. Darüber hinaus werden jeweils bestimmte kognitive, voluntative und affektive Kräfte des Redners 
an die des Hörers angemessen angepasst (vgl. ebd., S. 135f.). Vom wissenschaftlichen Interesse sind im Einzelnen verbale, para- und nonverbale Ausdruckmittel bzw. -verhalten, wie etwa die sprachliche Gestaltung von Mitteilungen, Aussprache und Prosodie, Mimik, Gestik, Blick- und proxemisches Verhalten etc.

Die Rhetorische Kommunikation als kommunikatives Sprech- und Hörhandeln, das grundlegend durch intentionale Finalität gekennzeichnet ist (vgl. Forster 1997, S. 57), hat allerdings nicht nur das Auslösen von Sprechhandlungen zum Ziel. Gleichzeitig kommt es auf die Veränderung sozialer Praxis durch gemeinsames Handeln an (vgl. Geißner 1981, S. 159). Dies kann durch wechselseitige intentionale Verständigungshandlung im und durch Gespräch geschehen. Die Mittel der Téchne in antiker griechischer und römischer Auffassung stellen somit lediglich einen Teilaspekt der modernen Rhetorik dar. Vielmehr ist hier das Problem zentral, ,wie die Menschen mittels mündlicher Kommunikation gemeinsame Handlungsziele erkennen, verfolgen und umsetzen oder was sie daran hindert" (Bose et al. 2009, S. 27).

Das eigentliche Ziel der Rhetorischen Kommunikation als Fachgebiet besteht demnach in der ,Analyse und Optimierung der interpersonellen Sprechkommunikation“ (Neuber 2013, S. 103). Diese Bemühungen sollten zur Vergrößerung des Bereichs des freien und bewussten kommunikativen Handelns führen (vgl. Bose et al. 2009, S. 27).

\subsection{Rhetorisch/Rhetorizität}

In Bezug auf das hier zugrunde gelegte Verständnis der Rhetorischen Kommunikation weist der Begriff des Rhetorischen zwei Spezifika auf. Zum einen ist damit Gutenberg zufolge die jeweilige Intentionalität von Sprechhandlungen gemeint, „die außer der Zweckabsicht (Zielintention) auch die Zweck-Mittel-Relation (Mittelintention) einschließt“ (1994, S. 435) sowie „alle auf ein Handlungsziel hin funktionalisierbaren Faktoren der Sprech-Hör-Situation umfaßt (Partnerintention, Situationsintention)“ (ebd.). Zum anderen bedeutet rhetorisch „denjenigen intentionalen Zugriff auf Ziele, Mittel und Situationsdeterminanten, der auf einer methodischen Reflexion beruht, die aufgrund einer didaktischen Anleitung zustandekommt" (ebd., S. 436). In praktischer Hinsicht handelt es sich hier um "eine theoriegeleitete Methodik einer didaktisch orientierten Theorie" (ebd.) und nicht um eine Technik bzw. Kunst schönen Redens. Zusammenfassend beinhaltet der Begriff des Rhetorischen gegenseitiges Beziehen der Kommunikationspartner aufeinander, Streben nach Zielen, die bewusste Wahl aus sprachlichem und sprecherischem wie auch nonverbalem Inventar, bewusstes Reflektieren kommunikativer Abläufe sowie Antizipation von Wirkungen des Gesagten bzw. des Gemeinten (vgl. Forster 1997, S. 45). 
Mit Rhetorizität übertragener Signale wird dementsprechend auf deren Intentionalität/Absichtlichkeit, Sinn- und Zweckorientiertheit, soziale Prägung etc. Bezug genommen. Zugleich ist Rhetorizität „nicht mehr und nicht weniger als umfassende bewußte kommunikative Handlungsfähigkeit" (Bose et al. 2009, S. 27) im Sinne einer Kompetenz aufzufassen (vgl. auch ebd., S. 23). Im Hinblick auf die bewusste Vermittlung dieser Kompetenz kommt es schließlich auf die Erziehung zur kritischen Mündigkeit im Sinne von Geißner an. Diese drückt sich in der Fähigkeit aus, sich zu äußern, Fragen zu stellen, zu akzeptieren, dass zum Lösen von Problemen Streit und Kooperation gehört (vgl. Geißner 1986a, S. 16). Damit werden Gesprächs-, Kritik-, Konflikt- und Verantwortungsfähigkeit als Prozesskategorien impliziert (vgl. ebd., S. 157; auch S. 203ff.).

\subsection{Rhetorische Kommunikation und der Wirkungsaspekt}

Im Kontext der Rhetorischen Kommunikation ist schließlich auf den bereits in der Antike bedeutenden Wirkungsaspekt zu verweisen. Aus wissenschaftstheoretischer sowie empirischer Perspektive wird diesbezüglich u.a. der Frage nachgegangen, welche Wirkungen mit Hilfe welcher Mittel durch das Zusammenwirken von Sprechenden und Hörenden angestrebt und konstituiert werden. Dies scheint ebenfalls in didaktischer Hinsicht bedeutend zu sein.

Die jeweils entstehenden (intendierten und/oder tatsächlichen) Wirkungen sind dabei sowohl am äußeren (materiellen) als auch inneren (gedanklichen) Handeln der jeweiligen Interaktionsakteure erkennbar (vgl. Bose et al. 2009, S. 27). Die Analyse von Rhetorizität übertragener Zeichen in den jeweils ablaufenden Kommunikationsprozessen ist folglich vor dem Hintergrund anderer situativer, kultureller etc. Faktoren auf ihre Wirkung hin höchst relevant (vgl. Mönnich 2004, S. 111). Von Interesse ist hier u.a. die Kenntnis darüber, auf der Basis welcher Zeichen die Kommunikationspartner einander als sachkompetent, kooperativ, engagiert, zielbewusst etc. betrachten.

\section{Kommunikation, Intentionalität und Kooperativität}

\subsection{Das Kommunikationsverständnis aus rhetorischer Sicht}

Im Hinblick auf die Rhetorizität der zwischenmenschlichen Verständigungsabläufe lässt sich der Begriff der Kommunikation keinesfalls auf technisch „Message“bestimmte Begrifflichkeiten einschränken. Denn bei der Kommunikation kommt es nicht nur auf die Übermittlung von Informationen bzw. deren Austausch an, sondern zugleich auch auf die Erreichung neuer Bewusstheitszustände, sprich: Wirkungen. Vor diesem Hintergrund wird in dem vorliegenden Beitrag von einer 
differenzierten Verwendung des Kommunikations-Begriffs im Sinne von Bartsch (2001, S. 31ff.) ausgegangen. Das hier aktuelle Kommunikationsverständnis soll in verschiedenen Zusätzen, wie etwa zwischenmenschliche, persönliche, faceto-face-Kommunikation, virtuell wechselseitige, verständigungs- bzw. konsensorientierte Kommunikation etc., erkennbar werden. Unter Kommunikation wird zusammenfassend ,das auf Verständigung zielende sprachliche und nicht-sprachliche zeichenhafte Handeln von Menschen“ (Becker-Mrotzek/Meier 2002, S. 19) verstanden. Dabei ist Verständigung in einem weiten Sinne ,als das Herstellen gemeinsamer Bedeutung für interaktiv realisierte Ziele“ (ebd.) aufzufassen. Demnach gilt Kommunikation zwischen Sprechern und Hörern als ständiger aufeinander bezogener „Interaktions- und Interpretationsprozeß“" (Selting 1997, S. 10). Das sprachliche Handeln stellt somit „eine interaktive Leistung“ (ebd.) dar, an der beide Kommunikationspartner (Sprecher und Rezipient) beteiligt sind. Im Sinne der Dialogizität der Rhetorischen Kommunikation trifft das ebenfalls für Rede als rhetorische Gattung zu.

\subsection{Der Zusammenhang zwischen Intentionalität und Kooperation}

Intentionalität und Kooperation als Merkmale der Rhetorischen Kommunikation stehen in einem besonders engen Zusammenhang, indem die Absicht des Sprechers ,die Kontaktherstellung zum Hörer [ist] mit dem Ziel, dass die Nachricht (intentionsadäquat) verstanden und der Sprecher selbst als Persönlichkeit (und in seiner gegenwärtigen Rolle) akzeptiert wird“ (Reinke 2008, S. 19). Der Kooperationswille der beiden Beteiligten stellt eine wesentliche Voraussetzung für eine funktionierende Kommunikation dar. Das sprachphilosophisch begründete Kooperationsprinzip von Grice - „Make your conversational contribution such as is required, at the stage at which it occurs, by the accepted purpose or direction of the talk exchange in which you are engaged" $(1975$, S. 45) - gilt somit als unerlässliche Bedingung für die Gewährleistung eines „maximal effektiven Informationsaustausche[s]" (ebd., S. 47), auch wenn die Identität der Sinnintention des Äußernden mit der des Hörers, u.a. auf der Grundlage bestimmter Erwartungsgewissheiten sowie bestimmter gesellschaftlicher Normen, nicht automatisch gesichert ist. Unterstützend soll dabei das Einhalten der sog. Konversationsmaximen sein: Maxime der Quantität bzgl. der Anteile an Informativität des jeweiligen Beitrags, der Qualität bzgl. der Wahrhaftigkeit/Wahrheit, der Relation bzgl. der Relevanz des sprachlich Formulierten und nicht zuletzt der Modalität bzgl. der Klarheit und Verständlichkeit sprachlicher Beiträge. Trotz gewisser Mängel dieser Interaktionspostulate (u.a. hinsichtlich der nicht eindeutigen Abgrenzung untereinander) sind sie als Leitlinien für das Partnerverhalten in Gesprächen offenkundig für alle Formen des Kommunizierens relevant (vgl. Heinemann/Heinemann 2002, S. 27f.; Fiehler 1999, S. 52ff.). 
Als kommunikationsfördernd können in analoger Weise ebenfalls Geltungsansprüche an eine ideale Sprechsituation innerhalb des kommunikativen Handelns von Habermas (1981) angesehen werden, deren Erfüllung die gegenseitige Kommunikation mit dem Ziel einer Verständigung/Übereinstimmung (eines Konsens) sichert. Die von Habermas konzipierten vier Diskursregeln umfassen die Wahrhaftigkeit im Selbst-Ausdruck (d.h. die Aufrichtigkeit), die Richtigkeit im appellativen Gebrauch der interpersonalen Codes (m.a.W. die Richtigkeit der Norm), die Wahrheit des sachlich Dargestellten (d.h. die Wahrheit in Bezug auf den propositionalen Gehalt der Sprachakte) wie auch die Verständlichkeit der verwendeten Zeichen.

Aufbauend auf den Grundgedanken von partnerschaftlich orientierten Handlungsweisen in Rede und Gespräch, einschließlich aktiven Zuhörens, des Perspektivwechsels und einer sachlichen und personalen Beziehungsarbeit, ist der Kooperativitätsgrundsatz nicht zuletzt explizit in den Ansatz der kooperativen Rhetorik einbezogen worden (vgl. Bartsch 1990, S. 37ff.). Kooperation stellt dabei nicht nur ein ethisches Postulat dar. Sie ist vielmehr eine Notwendigkeit, denn Kommunikation funktioniert nur kooperativ: Wenn der Zuhörer nicht will, kann der Redner sein Ziel nicht erreichen.

\section{Sprechen aus handlungstheoretischer Perspektive}

\subsection{Die Auffassung von Sprechhandlung aus Sicht der Rhetorik}

Aus handlungstheoretischer Perspektive ist Sprechen selbst als Handeln durch Sprechen und im Sprechen aufzufassen, wobei es auf eine bestimmte Art, also einen gewissen Modus des Handelns, ankommt (vgl. Bühler 1999/1934, S. 52). Mit Sprechen wird ein soziales Verhältnis impliziert, das über eine physiologischphysikalische, individual-psychologische Handlung des Sagens, über einen konkreten Akt der Sprachverwendung, die Lautbildung und die Vorstellungs- und Lautverknüpfung im Sinne von de Saussure hinausgeht. Demzufolge ist nicht die Sprache im Sinne eines in seiner Struktur beschreibbaren Zeichensystems als ein sozialer Tatbestand zu erfassen, sondern das Handeln durch Sprechen. Die Sprache stellt lediglich ein Instrument dar, mit dem Sprechhandlungen als primäre soziale Kategorien vom intentionalen Charakter als Handlungen durch Sprechen im Sinne von Bühler (1999/1934, S.46) vollzogen werden. In einzelnen intentionalen Sprechhandlungen wird also jeweils die Sprache als ideales Gebilde, also als (Zeichen--)Gerät, konkret realisiert (vgl. ebd., S. 48). Nichtsdestotrotz ist auch die Dialektik von Sprache und Sprechen zu betonen: So wenig wie es Sprechen ohne Sprache gibt, so wenig funktioniert Sprache ohne Sprechen (vgl. Geißner 1986b, S. 50). 
Vor dem Hintergrund der kommunikationskonstituierenden Dialogizität rhetorischer Prozesse ist jedoch das Bühlersche Verständnis von Sprechhandlung laut Geißner auf das Miteinandersprechen zwangsläufig zu erweitern. Denn das Wesen von Sprechhandlungen besteht vielmehr darin, dass Menschen miteinander über etwas in einer Sprache sprechen (vgl. Geißner 1986a, S. 95). Das Miteinandersprechen, das in jeglichem Miteinandersein von Menschen - vom Schweigen bis zur öffentlichen Rede - präsent ist, erfolgt jeweils ,am konkreten Ort zur konkreten geschichtlichen Zeit" (ders. 1986a, S. 93). Von daher ist jede Sprechhandlung als ,soziale Interaktion am genauen Punkt innerhalb der Raum-Zeit-Koordinaten eines beschreibbaren Handlungsfeldes" (ebd.) zu begreifen, die auf Verständigung mit Hilfe sprachlicher Zeichen und Symbole sowie auf deren Austausch bei verschiedensten Bedeutungen und verschiedensten Intentionen von Kommunikanten abzielt (vgl. ebd., S. 191). Aus der Wechselhaftigkeit im Prozess des Miteinandersprechens resultiert zugleich die zum Sprechen korrespondierende Tätigkeit des Hörens, so dass die Kommunikationskette aus Sprecher und Hörer besteht (vgl. auch Gutenberg 2001, S. 578). Dabei kann jeder der beiden Teilnehmer sprechen und hören, „wenn das ,mit" seinen Sinn behalten soll“" (Geißner 1986a, S. 95). Die jeweiligen sprecher- und hörerseitigen Vorerwartungen sind von gleichwertiger Bedeutung. Die beiden zueinander komplementären Tätigkeiten Sprechen und Hören/Verstehen gelten Geißner zufolge nicht zuletzt als Prozesse des intentionalen Handelns, die auf der höheren Beschreibungsebene als Sprechdenken und Hörverstehen bzw. als Sprech- und Hörhandeln beschreibbar sind (vgl. ders. 1981, 45; auch ebd., S. 165).

\subsection{Bestimmungsmerkmale des sprechsprachlichen Handeln}

Handeln ist Geißner zufolge durch folgende Bestimmungsmerkmale gekennzeichnet: Absichtlichkeit/Intentionalität und Kooperation/Kooperativität ${ }^{1}$ sowie Zweck- und Sinnorientiertheit, in der die jeweils Handelnden mit dem jeweiligen Handeln einen subjektiven Sinn konstituieren - nicht nur einfach aus der Struktur der Zeichen, sondern vielmehr in konkret ablaufenden Kommunikationsprozessen (vgl. Geißner 1981, S. 74). Für das jeweilige Handeln sind des Weiteren auch Perspektivität und Antizipierbarkeit als wichtige Voraussetzungen für die jeweilige Planung von Sprechhandlungen bestimmend, die dementsprechend einen Entwurfscharakter aufweisen. Die jeweiligen Vorentwürfe sind allerdings „keine totalen Neu-Erfindungen durch individuelle Subjekte, sondern es sind Wahlen aus bereits

${ }^{1}$ Kooperativität stellt eine Kategorie zur Bewertung von Kommunikationsverhalten durch die Kommunikationsbeteiligten selbst dar, indem sie im Verlaufs des Gesprächs oder danach „den Eindruck haben, daß sich andere Beteiligte oder sie selbst sich kooperativ verhalten haben“ (Fiehler 1999, S. 52). 
im gesellschaftlichen Prozeß ,eingeschliffenen Formen“"(Geißner ebd., S. 29), bei denen es sich um Handlungsmuster im Sinne von Rehbein handelt. Diese sind wiederum ,auf der Grundlage gesellschaftlicher Kooperation“ (Rehbein 1977, S. 90) durch Erprobung entstanden. Von besonderer Relevanz ist schließlich auch das Erwartungsmerkmal: Auf Grund gewisser Erwartungen ist jede Handlung nicht nur durch deren Gegenwart bzw. Nachgeschichte gekennzeichnet, sondern auch durch eine Vorgeschichte, so dass zwischen präkommunikativer, kommunikativer und postkommunikativer Phase von Handeln zu unterscheiden ist.

Die Sinnkonstitution erfolgt vor dem Hintergrund situativer, personaler, sprachlicher, formativer und nicht zuletzt leibhafter Faktoren. Im Einzelnen kommt es auf die situative Steuerung von Sprechhandlungen an, die der linguistisch-pragmatisch begründeten Einbettung der Sprechhandlungen in einen jeweils größeren situativen Kontext entspricht. Die jegliche Äußerung und die Situation, in der sie vollzogen wird, stellen dabei eine Einheit vom reziproken Charakter dar. Die Situation wirkt einerseits auf die Sprachäußerung, sie wird aber zugleich vom sprachlichen Geschehen mit definiert. Ferner handelt es sich um die wechselseitige Bindung der miteinander sprechenden Personen als Sprecher und Hörer (mit Raum- und Zeiterfahrungen), die Dialektik von Sprache (Zeichensystem) und Sprechen (Sprechausdruck). Hinzu kommt das aus dem Miteinandersprechen resultierende Streben nach Gesprächsfähigkeit unter Berücksichtigung aller Sprachebenen: der phonologischen, morphologischen, syntaktischen und semantischen Formbestimmtheit (Musterhaftigkeit) von Sprechhandlungen. Insgesamt handelt es sich hier also um ein Faktorengefüge, das sich in gegebenen Sprechhandlungen ausdrückt und im Rahmen dessen die gegebenen sozialen Rollen in die entsprechenden Sprechrollen umgesetzt werden. Dabei wird jeweils ein konkretes, gruppenspezifisches Sprechverhalten erwartet (vgl. Geißner 1986a, S. 98; auch Neuber 2013, S. 104f.). Dies entspricht der Theorie der sozialen Rollen von Dahrendorf (2006), nach der jegliches Handeln immer in (durch Beruf, Familie, Alter, Geschlecht determinierte) Rollen eingebunden ist (vgl. auch Neuber ebd.).

\subsection{Ganzheitliche Betrachtung der gesprochenen Sprache}

In dem vorliegenden Beitrag wird, wie einleitend erwähnt, von der ganzheitlichen Betrachtung der gesprochenen Sprache ausgegangen. Das Material der Kommunikation umfasst folglich nicht nur Worte, sondern auch alle paralinguistischen Phänomene (Stimme/Stimmqualität, Segmentalia, Suprasegmentalia, ferner auch Seufzen, Räuspern etc.) sowie Körperausdruck (Mimik, Gestik, Proxemik und Kinesik) (vgl. Watzlawick et al. 1974, S. 51; Argyle 1992, S. 150). In diesem Sinne kommt es jeweils zum gegenseitigen Zusammenspiel von Sprach- und Sprechstil. Erst in diesem Zusammenwirken von Was und Wie des Sprechens, 
sowie bei der Verankerung mündlicher Verständigungsprozesse im nonverbalen Bereich, wird der jeweilige Sinn des Gesprochenen konstituiert (vgl. Geißner 1986a, S. 109). Die gesprochene Sprache erfüllt dabei nicht nur linguistische, sondern auch zugleich kommunikative Funktionen, denn mit jeder Äußerung werden auch Informationen über die Sprechperson vermittelt. D.h., in der Kommunikation kommt es ebenfalls auf nichtsprachliche Äußerungsmöglichkeiten an - als Elemente ,des Verhaltens, der Erscheinung usw. eines Organismus, das von den Sinnesorganen eines zweiten Organismus wahrgenommen wird und dessen Verhalten beeinflußt" (Argyle 1992, S. 14). So wirken in der direkten Kommunikation jeweils verschiedene Kommunikationskanäle zusammen, wie etwa der taktile (Körperberührung, Streicheln), der olfaktorische (Riechen von Körpergeruch, Parfüm), der thermale (Spüren von Körperwärme) und der gustatorische Kanal (Geschmacksempfindungen) (vgl. Scherer 1970, S. 3f.). Die komplexen Wechselwirkungen verschiedener Kommunikationsebenen stellen dabei - trotz deutlicher Trennungslinie ,entlang der Unterscheidung Stimmgebundenheit (akustische Wahrnehmung) vs. Leibgebundenheit (optische Wahrnehmung)“ (Fiehler et al. 2004, S. 67) - einen Normalfall der in der Mündlichkeit ablaufenden sozialen Interaktionen dar. Die Ziehung einer Grenze zwischen Linguistik, Para- und Extralinguistik ist folglich kaum möglich. Somit läuft auch die Beurteilung der Gesprochenen Sprache in der face-to-face-Kommunikation zugleich unter dem auditiven und visuellen Aspekt ab (vgl. z.B. Potapova 2003, S. 275). Die Phänomene des Körperausdrucks ebenso wie die des Sprechausdrucks, die ein untrennbarer Bestandteil der direkt ablaufenden Kommunikationsprozesse sind bzw. an diesen Prozessen jeweils einen festen Anteil haben, stellen deshalb keineswegs nur Zusätze zum Sprachlichen dar.

\section{Sprechwissenschaftlich relevante Kommunikationsansätze}

\subsection{Geißners Gesprächsmodell}

Im Kern des Gesprächsmodells von Geißner (vgl. 1981, S. 35ff.) steht das Phänomen des Miteinandersprechens, welches auch für verschiedene sprechwissenschaftlich angelegte Beschreibungen von Prozessen mündlicher Kommunikation maßgebend ist (vgl. z.B. Forster 1997, S. 19f.). Nach diesem Modell bestimmt das Miteinandersprechen den ,formalen Verlauf und zugleich die Prozeßhaftigkeit von ,Gespräch““ (Geißner ebd., S. 37). Einer und (wenigstens) ein anderer sprechen miteinander, d.h. sie sprechen formal nicht nacheinander, sondern im Wechsel (vgl. auch ders. 1986a, S. 94). Als gesprächskonstituierend gilt allerdings nicht nur die Wechselhaftigkeit des Redestroms. Für die Konstitution von Gesprächen ist zugleich auch Fragen ausschlaggebend, da Gespräche jeweils durch 
fragend-antwortendes Miteinandersprechen entstehen. Hier handelt es sich um akustische Verständlichkeit sowie der Verständigung halber um nicht-gleichzeitiges Sprechen (vgl. Geißner 1981, S. 37). Insgesamt gilt das Gespräch als „Prototyp kommunikativen Handelns“ (ebd., S. 46). Es ist ,als mündliche Kommunikation die intentionale, wechselseitige Verständigungshandlung mit dem Ziel, etwas zur gemeinsamen Sache zu machen, bzw. etwas gemeinsam zur Sache zu machen" (ebd., S. 45). Es lässt sich somit als Prozess des willentlich begonnenen, intentionalen, wechselseitigen Sprechens und Hörens bestimmen. Dabei kommt es zu einer Verständigung im weiteren Sinn, sowohl über eine Koordination als auch über einen Konflikt: [S]ofern (...) das Gespräch nicht verweigert oder abgebrochen wird, bleibt es - selbst unter verzerrenden Bedingungen - ein Prozeß gesellschaftlicher Kooperation“(ebd., S. 44; vgl. auch Slembek 1986, S. 120f.).

\subsection{Geißners Situationsmodell}

Das sog. (Sprech-)Situationsmodell von Geißner (1981, S. 72ff.) stellt aus Sicht der Rhetorischen Kommunikation ein durchaus geeignetes Instrument für die Analyse der jeweiligen (Sprech-)Situationen dar. Mit dessen Hilfe lässt sich eine Reihe von Fragen jeweils unter zwei Gesichtspunkten betrachten: der Sprecherabsicht und -erwartung vs. Hörerabsicht und -erwartung. Zentral stehend sind dabei die Kommunikationsbeteiligten (wer? und mit wem?) in den jeweils sozial determinierten Sprechrollen sowie der Kommunikationsgegenstand (worüber?), die allerdings aus dem Gesagten und den Metadaten (Informationen über die Beteiligten) nur unmittelbar abgeleitet werden können. $\mathrm{Zu}$ rahmenden Faktoren gehören ferner Zeit (wann?), Ort (wo?) wie auch Anlass (warum?) und Ziel (wozu?) (vgl. auch ders. 1986a, S. 192). Geradezu die zwei letztgenannten Faktoren erlauben Neuber zufolge festzustellen, „ob überhaupt rhetorische Kommunikation vorliegt, da aus der Relation von Anlass und Ziel erklärbar wird, wie die Sprechsituation bezüglich ihrer gesellschaftlichen Relevanz einzuordnen ist und ob deklariert zielgeleitet kommuniziert wird“" (2013, S. 105). Die Kategorie der Sprechsituation gilt insgesamt ,als Bedingungsgefüge gesellschaftlicher und individueller Voraussetzungen“ (Lepschy 2002, S. 51), wodurch sie auch kein statischer Zustand ist. Vielmehr unterliegt sie dynamischen Veränderungen.

Im Hinblick auf die oben erläuterte Erweiterung der Sprechhandlung um die Konzeption des Miteinandersprechens ist das Situationsmodell Geißner zufolge als eine legitime Erweiterung des Organon-Modells von Bühler (1999, S. 24ff.) anzusehen, das ,ein aus (...) vorwiegend psychologischen Vorgriffen erklärbares Sprecher-Modell; ein Modell des ,Sprechens zu jemandem', aber nicht des ,Miteinandersprechens"“ (Geißner 1986a, S. 96) darstellt und in dieser Form für die Beschreibung von Sprechhandlungen als unzureichend erscheint. Denn es bleibt hier die Frage unberücksichtigt, was in der gegebenen Sprechsituation mit dem 
Hörer passiert, ob er völlig passiv ist während ihm etwas kundgetan, etwas dargestellt bzw. wenn an ihn appelliert wird oder ob er in alle drei Grundfunktionen (die Ausdrucks-, Darstellungs- und Appellfunktion) einwirkt. Diesbezüglich ist mithin nicht nur von Sprechsituation auszugehen. Von Relevanz ist ebenfalls der Begriff der Hörsituation als „Gesamtheit aller vorausliegenden psychischen Erlebnisse bis zum Augenblick des Hörens, die irgend mit dem Gehörten in Beziehung stehen können“"(Drach 1926, S. 138f.). Dabei sind Sprech-und Hörsituation komplementär aufeinander bezogen, ebenso wie Sprechdenken und Hörverstehen (vgl. auch Forster 1997, S. 300).

\subsection{Die Totalität des Sprechens aus Gutenbergs Sicht. Sprechtätigkeit}

Ausgehend von der Tätigkeitstheorie von Leont’ev (vgl. u.a. 1971) zielt auch Gutenberg auf eine integrale Erfassung von Prozessen der mündlichen Kommunikation als Gesamtkomplex ab. Als zentrale Kategorie gilt bei ihm Dialog. In Gutenbergs Konzeption werden ,alle [in einem Außenkreis stehenden] Dimensionen der Totalität des Miteinandersprechens“ (1998, S.8), d.h. „Intersubjektivität, Personalität, Leiblichkeit, Materialität, Sozialität, Kulturalität und Sprachlichkeit im Spannungsfeld von Mündlichkeit und Schriftlichkeit, Medialität und raumzeitlicher Unmittelbarkeit“ (ebd.) vermittelt. Dabei kann das Sprechen ,als eine und in einer unterbrochenen Folge von Gesprächen und Reden“" (ders. 1981, S. 25) und somit als Teil der Gesamtäußerungen der Menschen aus historisch-gesellschaftlicher Entwicklungsperspektive begriffen werden. Tätigkeiten, deren Motiv und Ziel den jeweils agierenden Subjekten bewusst werden können, aber nicht unbedingt müssen, bedeuten in diesem Verständnis die Gesamtheit menschlichsozialen Tuns (ders. 1994, S. 86f.). Das (Miteinander)Sprechen stellt dabei eine besondere Tätigkeit dar, d.h. es gibt die Sprechtätigkeit als solche nicht, sondern es handelt sich um ein System von Sprechhandlungen, ,die zu irgendeiner Tätigkeit gehören“ (Leont'ev 1971, S. 31). Kurzum: Die Sprechtätigkeit ist eine Tätigkeit, die Sprache benutzt und produziert, der Mensch kann aber mit dem Sprechen allein nichts anfangen. Sprechen ist Leont'ev zufolge nicht Selbstzweck, sondern Mittel und Werkzeug, das bei verschiedenen Arten von Tätigkeiten verschieden verwendet werden kann (vgl. Leont'ev ebd.). Die Sprechtätigkeit ist zugleich mit anderen Formen der menschlichen Lebensäußerungen verflochten, so dass von einem „vertikal und horizontal interdependenten Musternetz auszugehen“ (Gutenberg ebd., S. 88) ist. Dieses umfasst folgende Muster: sprechtätigkeitstranszendente, soziale (Schicht, Institution usw.), psychische (Affekte, Haltungen usw.), psychosoziale (Rolle, Situation usw.), physiologische (Körperbau, somatische Krankheiten), sprachliche (Phonemsystem, Grammatik) und physikalische (Raum, Zeit, Technik). Ferner gehören dazu Sprechhandlungsmuster der Makroebene wie Gespräch (Debatte, Beichte etc.), Rede (Ansprache, Standpauke, 
Vorlesung etc.) und der Mesoebene (Argumentation, Erzählung) sowie Sprechoperationsmuster der Mikroebene, auf denen komplexe Sprechhandlungen aufbauen (Sprechakte: Fragen, Behaupten etc.). Die einzelnen Muster sind jeweils auf eine komplexe Weise miteinander verwoben (vgl. ebd., S. 93f.; auch ders. 2001a, S. 69ff. und 82). Verkürzt lässt sich Sprechtätigkeit auffassen als „eine gleichzeitig dialogische, sprachliche, sozial-kulturelle, psychische, physische, physikalische, historische, formative Tätigkeit, die Sinn konstituiert und andere Tätigkeiten auslöst““(ders. 1998, S. 7).

\subsection{Das Johari-Fenster}

In der gesamten Diskussion um die Rhetorizität kommunikativer Prozesse sind auch weitere, darunter psychologische Kommunikationsansätze von Relevanz, die an die jeweilige Deutung der rhetorischen Kommunikationsabläufe heranzuziehen sind. Zunächst sei auf das sog. Johari-Fenster (vgl. Luft/Ingham 1955) verwiesen, das die Verhältnisse des Bewussten vs. des Unbewussten sowie die Darstellung unterschiedlicher Selbst- und Fremdeinschätzungen, die als Ursache für Kommunikationsstörungen angesehen werden, widerspiegelt. Das Hauptziel der rhetorischen Forschung wäre demnach, möglichst umfangreiche Bereiche des Sprechhandelns durch Reflexion und Bewusstheit für viele zugänglich zu machen (vgl. auch Neuber 2013, S. 106).

\subsection{Das Vier-Ohren-Modell}

Maßgebend für die Betrachtung der rhetorischen Kommunikationsprozesse ist ferner das sog. Vier-Ohren-Modell (das Vier-Seiten-Modell) von Schulz von Thun (1993/1977, S. 14 und 45) (Die vier Seiten einer Nachricht und Der vierohrige Empfänger) als Weiterentwicklung der Kommunikationsaxiome von Watzlawick (vgl. Watzlawick et al. 1974). Hier wird deutlich, dass in einer konkreten Nachricht nicht nur der gegebene Sachinhalt kommuniziert wird, sondern auch Signale über Beziehungen, Selbstoffenbarung sowie Appelle, die oftmals nicht ausgesprochen werden. Die o.g. vier Seiten einer Nachricht werden dann entsprechend mittels einer Reihe von Fragen (1. Was ist das für einer? Was ist mit ihm?; 2. Wie redet der eigentlich mit mir? Wen glaubt er vor sich zu haben?; 3. Wie ist der Sachverhalt zu verstehen?; 4. Was soll ich tun, denken, fühlen?) erfasst. Zugleich sind hier mögliche Missverständnisse in Kommunikationsprozessen zu berücksichtigen, die auf Grund unterschiedlicher Erfahrungen, Erwartungen sowie Vorurteile der Kommunikationspartner zustande kommen. Im Vordergrund steht die Frage nach Ursachen für potenzielle Kommunikationsverzerrungen, die verbale para- und nonverbale Signalisation sowie ,die Frage nach der Wirkung im Rahmen der Rhetorizität“" (Neuber 2013, S. 107). 


\subsection{Das Modell der Trivialen und Nichttrivialen Maschine}

Schließlich ist an dieser Stelle das (in Konstruktivismus und Systemtheorie grundlegende) Modell der Trivialen und Nichtrivialen Maschine von Foerster (1997) zu nennen, nach dem zwischen analytisch bestimmbaren, vergangenheitsunabhängigen und voraussagbaren (TM) und analytisch unbestimmbaren, vergangenheitsabhängigen und unvoraussagbaren (NTM) Größen unterschieden wird (vgl. Neuber 2013, S. 108). Während die triviale Maschine laut Meyer (2011, S. 40ff.) ein einfaches und durchschaubares Konstrukt darstellt, das auf einen bestimmten Input (Reiz, Ursache) immer mit einem bestimmten Output (Reaktion, Wirkung) reagiert, ist die nicht triviale Maschine „viel unberechenbarer“ (ebd., S. 41) trotz ihrer scheinbaren Ähnlichkeit zur trivialen Maschine. Die Output-Reaktion wird hier zugleich durch einen (sich im Innern der Maschine befindenden) Zustand beeinflusst, der aber zugleich auch sich selbst beeinflusst, so dass die Funktionsweise der nicht-trivialen Maschine einer zirkulären, rückbezüglichen Kausalität unterliegt und somit nicht bestimmbar ist (vgl. ebd.). Ein gegebenes Eingangssymbol werde nicht dasselbe Ausgangssymbol hervorrufen, da die Operationen der Maschine von den Operationen ihrer Vergangenheit abhängig seien (vgl. Foerster 1997, S. 39; zit. nach Meyer ebd.). Diesbezüglich soll Meyer zufolge der Versuchung widerstanden werden, „das Problem der Nicht-Trivialität (weiterhin) zu ignorieren oder interpersonelle Kommunikation zu trivialisieren“ (ebd., S. 45). Vielmehr gilt es zu versuchen, „eine Epistemologie der Nicht-Trivialität zu entwickeln“ (ebd.). Für die rhetorische Lehre könnte dies bedeuten, ,sich endgültig von ,richtig-falsch'-Kategorien zu verabschieden und im Feedback noch stärker Subjektivität und aktuelle Situationsbezogenheit zu betonen“" (ebd.).

\section{Zusammenfassende Bemerkungen. Rhetorische Kommunikation aus praxisbezogener Sicht}

Für die Betrachtung der Rhetorischen Kommunikation sind insgesamt folgende Kernaspekte von Belang:

- das grundlegende kommunikativ-dialogische Verständnis sozialer Interaktionen;

- das auf die Konzeption von Miteinandersprechen aufbauende Sprechsituationsmodell, verkürzt in der Formel ,Wer spricht mit wem worüber wann und wo?";

- der intentionale Handlungscharakter von Sprechhandlungen, der im handlungsauslösenden Sprechdenken und Hörverstehen in verschiedenen Redeund Gesprächsformen zum Ausdruck kommt;

- der Kooperatiossprinzip;

- der Aspekt von Verständlichkeit sprechsprachlicher Kommunikation; 
- das Wirkungsphänomen und das im Zusammenhang damit stehende sowohl bewusste als auch unbewusste Handeln bzw. Verhalten - entsprechend der Maxime von Watzlawick et al. (1974, S. 51), man könne nicht nicht kommunizieren;

- die Situations- und Kontextgebundenheit kommunikativer Abläufe, d.h. die Totalität des Sprechens - dessen Einfügung in ein Netz unterschiedlicher Interdependenzen und dessen Ablauf vor dem Hintergrund sprachlicher, physischer und physikalischer/materieller (sprecherischer/körperlicher), psychosozialer, physiologischer, sozialer sowie kultur- und gesellschaftsgeschichtlicher, personell-intersubjektiver Dimensionen (die Multimodalität sprechsprachlicher Verständigungsprozesse).

Die Problemstellungen der Rhetorischen Kommunikation kommen im Hinblick auf den angestrebten Abbau möglicher Kommunikationsbarrieren vor allem mit denen des fremdsprachlichen Lehrens und Lernens am ehesten zusammen (vgl. Teuchert 1992, S. 67). Aus der Perspektive der Fremdsprachenvermittlung stellt die oben beleuchtete Auffassung der Rhetorischen Kommunikation eine hilfreiche Grundlage für die Thematisierung der rhetorischen Aspekte im Fremdsprachenunterricht dar. Damit sind nicht nur rein verbal-lautliche sowie para- und nonverbale Komponenten der gesprochenen Sprache gemeint. Vielmehr handelt es sich auch um all diejenigen Phänomene, die eine effektive Kommunikation weitgehend ermöglichen, wie etwa Intentionalität, Kooperativität, Verständlichkeit, Multimodalität der kommunikativen Bedingungen etc. Die jeweils gegebene Rhetorizität hängt darüber hinaus von einer Reihe weiterer Faktoren ab, wie etwa soziale Rollen der Kommunikanten und deren Statusverhältnisse, Erwartungsansprüche und deren (Nicht-)Erfüllung, Nachwirkungen vorausgehender Kommunikationsereignisse etc. (vgl. Hirschfeld/ Neuber 2010, S. 50). Ohne deren Bewusstmachung wäre die zwischenmenschliche Kommunikation tatsächlich auf eine bloße Übertragung von Informationen reduziert, was aber in der sprechsprachlichen Wirklichkeit keinesfalls der Fall ist.

\section{LITERATURVERZEICHNIS}

Argyle M. (1992), Körpersprache und Kommunikation, (= Reihe Innovative Psychotherapie und Humanwissenschaften, Bd. 5), Paderborn.

Bartsch E. (1990), Grundlinien einer „,Kooperativen Rhetorik“. In: Geißner H. (Hrsg.): Ermunterung zur Freiheit. Rhetorik und Erwachsenenbildung. Festschrift für Ilse Schweinsberg zur Vollendung ihres 70. Lebensjahres, (= Sprache und Sprechen Band 23/24), Frankfurt/M., S. 37-49.

Bartsch E. (2001), Was heißt eigentlich „Kommunikation“? Vorschläge zur differenzierten Verwendung des Begriffs, In: Allhoff D.-W. (Hrsg.): Schlüsselkompetenz Mündliche Kommunikation, München, S. 31-48.

Becker-Mrotzek M./Meyer Ch. (2002), Arbeitsweisen und Standardverfahren der Angewandten Diskursforschung. In: Brünner G., Fiehler R., Kindt W. (Hrsg.): Angewandte Diskursforschung, Band 1: Grundlagen und Beispielanalysen, Radolfzell, S. 18-45. 
Bose I., Hirschfeld U., Neuber B. (2009), Verständlichkeit und barrierefreie Kommunikation aus sprechwissenschaftlicher Sicht. In: Antos G. (Hrsg.): Rhetorik und Verständlichkeit, Band 28 Rhetorik, Ein internationales Jahrbuch, Tübingen, S. 21-33.

Bühler K. (1999/1934), Sprachtheorie. Die Darstellungsfunktion der Sprache. Mit einem Geleitwort von Friedrich Kainz, 3. Aufl., Stuttgart.

Bußmann H. (1990), Lexikon der Sprachwissenschaft, Stuttgart.

Dahrendorf R. (2006/1958), Homo sociologicus. Ein Versuch zur Geschichte, Bedeutung und Kritik der Kategorie der sozialen Rollen, 16. Aufl., Wiesbaden.

Drach E. (1926), Die redenden Künste, Leipzig.

Fiehler R. (1999), Was tut man, wenn man ,kooperativ' ist? Eine gesprächsanalytische Explikation der Konzepte ,Kooperation ' und ,Kooperativität'. In: Mönnich A./Jaskolski E. W. (Hrsg.): Kooperation in der Kommunikation. Festschrift für Elmar Bartsch, München, Basel, S. 52-59.

Fiehler R., Barden B., Elstermann M., Kraft B. (2004), Eigenschaften gesprochener Sprache, (= Studien zur Deutschen Sprache. Forschungen des Instituts für Deutsche Sprache. Herausgegeben von Ulrike Haß-Zumkehr, Werner Kallmeyer und Ulrich Waßner, Bd. 30), Tübingen.

Foerster H. v. (1997), Abbau und Aufbau. In: Simon H. v. (Hrsg.): Lebende Systeme. Wirklichkeitskonstruktionen in der systemischen Therapie, Frankfurt/M., S. 32-51.

Forster R. (1997), Mündliche Kommunikation in Deutsch als Fremdsprache: Gespräch und Rede, (=Sprechen \& Verstehen. Schriften zur Sprechwissenschaft und Sprecherziehung, Bd. 12), St. Ingbert.

Frank-Böhringer B. (1963), Rhetorische Kommunikation, Quickborn.

Geißner H. (1981), Sprechwissenschaft. Theorie der mündlichen Kommunikation, Königstein.

Geißner H. (1986a), Rhetorik und politische Bildung, Frankfurt/ M.

Geißner H. (1986b), Sprecherziehung. Didaktik und Methodik der mündlichen Kommunikation, 2. Aufl., Frankfurt/M.

Grice P. (1975), Logic and conversation. In: Cole P./Morgan J. (Hrsg.): Syntax and Semantics 3: Speech Acts, New York, S. 41-58.

Gutenberg N. (1994), Grundlagenstudien zu Sprechwissenschaft und Sprecherziehung. Kategorien - Systematik - Programm, (= Göppinger Arbeiten zur Germanistik hrsg. von Ulrich Müller, Franz Hundsnurscher und Cornelius Sommer, Nr. 615), Göppingen.

Gutenberg N. (1998), Einzelstudien zu Sprechwissenschaft und Sprecherziehung. Arbeiten in Teilfeldern, (= Göppinger Arbeiten zur Germanistik hrsg. von Ulrich Müller, Franz Hundsnurscher und Cornelius Sommer, Nr. 616), Göppingen.

Gutenberg N. (2001), Einführung in die Sprechwissenschaft und Sprecherziehung, Frankfurt/M.

Habermas J. (1981), Theorie des kommunikativen Handelns, Frankfurt/M.

Heinemann M./Heinemann W. (2002), Grundlagen der Textlinguistik: Interaktion - Text - Diskurs, (= Reihe Germanistische Linguistik), Tübingen.

Hirschfeld U./Neuber B. (2010), Methodische Überlegungen zur Untersuchung phonetischer und rhetorischer Parameter im interkulturllen Wissenschaftsdiskurs. In: Sprechen, H. 49, S. 45-55.

Leont'ev, A. A. (1971), Sprache - Sprechen - Sprechtätigkeit, Stuttgart.

Lepschy A. (2002), Lehr-und Lernmethoden zur Entwicklung von Gesprächsfähigkeit, In: Brünner G./Fiehler R./Kindt W. (Hrsg.): Angewandte Diskursforschung, Bd. 2: Methoden und Anwendungsbereiche, Radolfzell, S. 50-71.

Luft J./Ingham H. (1955), The Johari Window, a graphic model for interpersonal relations, Los Angeles.

Meyer D. (2011), Alles nur Vermutung? Gedanken zur interpersonellen Kommunikation aus konstruktivistisch-systemischer Sicht. In: Bose I., Neuber B. (Hrsg.), Interpersonelle Kommunikation: Analyse und Optimierung, (= HSSP 39), Frankfurt/M., S. 39-46.

Mönnich A. (2004), Von der antiken Rhetorik zur Rhetorik der Gegenwart. In: Pabst-Weinschenk M. (Hrsg.), Grundlagen der Sprechwissenschaft und Sprecherziehung, München/Basel, S. 104-113. 
Neuber B. (2013), Definitionen und Grundvorstellungen (Rhetorische Kommunikation). In: Bose I./ Hirschfeld U./Neuber B./Stock E: Einführung in die Sprechwissenschaft, Tübingen, S. 101-108. Pabst-Weinschenk M. (2004), Rhetorische Kommunikation. In: Pabst-Weinschenk M. (Hrsg.): Grundlagen der Sprechwissenschaft und Sprecherziehung, München/Basel, S. 101-104.

Potapova R.K. (2003), Zur Kategorialität der Wahrnehmung der gesprochenen Fremdsprache. Linguistische, para- und extralinguistische Aspekte. In: Anders L. Ch., Hirschfeld U.: Sprechsprachliche Kommunikation. Probleme, Konflikte, Störungen (HSSP 12), Frankfurt/M., S. 271-280.

Rehbein J. (1977), Komplexes Handeln. Elemente zur Handlungstheorie der Sprache, Stuttgart.

Reinke K. (2008), Zur Wirkung phonetischer Mittel in sachlich intendierter Sprechweise bei Deutsch sprechenden Russen. (= Hallesche Schriften zur Sprechwissenschaft und Phonetik, Bd. 26), Frankfurt/M.

Selting M. (1997), Interaktionale Stilistik: Methodologische Aspekte der Analyse von Sprechstilen. In: Selting M., Sandig B.: Sprech- und Gesprächsstile, Berlin, New York, S. 9-43.

Scherer K.R. (1970), Non-verbale Kommunikation, Hamburg.

Schulz von Thun F. (1993/1977), Miteinander reden 1. Störungen und Klärungen. Allgemeine Psychologie der Kommunikation, Reinbek bei Hamburg.

Slembek E. (1986), Bilder als Impulse. Elemente der Gesprächserziehung im Unterricht Deutsch als Fremdsprache, In: Hermanns F. et al. (Hrsg.): Lehrmittel Deutsch 43, Neuchâtel, S. 119-134.

Watzlawick P., Beavin J. H., Jackson D.D. (1974), Menschliche Kommunikation. Formen Störungen. Paradoxien, 4. Aufl., Bern.

Beata Grzeszczakowska-Pawlikowska

\section{FROM RHETORIC TO RHETORICAL COMMUNICATION. A SHIFT IN TERM AND CONTENT}

(Summary)

The term Rhetorische Kommunikation (engl. rhetorical communication, vgl. McCroskey 1968 „An Introduction to Rhetorical Communication") was introduced into German-language scholarship by Brigitte Frank-Bohringer in her 1963 book of the same title and was subsequently firmly established by Helmut Geißner. It is currently used in a variety of contexts when the rhetorical dimensions of language are the focus of interest. It appears both in rhetoric manuals as well as in academic texts on the topic, including those with an empirical perspective. This contribution aims to illuminate the epistemological background to this terminology by focusing on a number of linguistically relevant communication approaches. Underlying this discussion is the differentiated use of the concept as outlined here, as well as a holistic view of spoken language, grounded in a theory of action. Finally, the practical value of the term Rhetorische Kommunikation, will be emphasized, underlining the fact that its content serves as a provable basis for the systematic and goal-oriented use of rhetoric as a theme in the teaching of German as a foreign language. The following discussion proceeds from the concept of Rhetorische Kommunikation.

Key words: rhetoric, rhetorical communication, spoken language, intentionality, cooperation, foreign language teaching. 